\title{
Occipital Horn of the Lateral Ventricle
}

National Cancer Institute

\section{Source}

National Cancer Institute. Occipital Horn of the Lateral Ventricle. NCI Thesaurus. Code C33195.

The part of the lateral ventricle that projects posteriorly into the occipital lobe. 